\title{
THE ANALYSIS OF THE INFLUENCE OF CORE COMPRESSION EFFECT ON THE DETERMINATION OF THE SHEAR MODULUS OF THE SANDWICH PANEL CORE
}

\author{
Jolanta Blaszczuk ${ }^{1}$, Zbigniew Pozorski $i^{2}$ \\ ${ }^{I}$ Institute of Mathematics, Czestochowa University of Technology, Poland \\ ${ }^{2}$ Institute of Structural Engineering, Poznan University of Technology, Poland \\ jolanta.blaszczuk@im.pcz.pl,zbigniew.pozorski@put.poznan.pl
}

\begin{abstract}
The paper concerns the problem of the influence of core compression on core shear modulus determination. Two experimental methods of shear modulus determination are discussed. These methods are based on the estimation of the relation between bending and shear effects. Using the ordinary sandwich panel theory. The appropriate examples of the core compression influence are presented and discussed.
\end{abstract}

\section{Introduction}

The intensive development of the sandwich structures came after the Second World War. The sandwiches turned out to be very attractive material for the military industry because of its main advantage - high load-bearing capacity at low self-weight.

The theory of sandwich structures was originated by Nicholas Hoff [1], Eric Reissner [2] and other scientists working for Forest Product Laboratories and NACA (NASA at present).

One of the most important aspects of the structural behaviour of the sandwich structure is the shear deformable core. Therefore, the determination of the proper shear modulus of the core is the crucial problem.

The problem of shear modulus determination was recognized as very important in everyday engineering practice, especially in high-technology industries [3]. The discussion about different test methods for shear modulus of sandwich cores applied in British and American Standards was presented in [4]. The paper [5] compares current test methods described in the European Standard (ES) [6]. The authors in [5] propose a new method of shear modulus determination based on measurements of angles of rotation. Assessment of shear modulus of the modern aluminum lattice truss core was presented in [7].

Two methods of shear modulus determination, based on the same ordinary sandwich theory, are recommended by the ES. Unfortunately, they yield different results. In the field of civil engineering the European Standard specifies requirements for sandwich panels applied as walls and roofs. 
This paper describes classical methods of shear modulus determination, compares the methods recommended by the ES and estimates the importance of the core compression effect. The resulting conclusions are confirmed experimentally.

\section{Methods of shear modulus determination}

There are three classical methods of shear modulus determination: direct shear, torsion and shear of the beam. The scheme of the first test called also double-lap test is illustrated in Figure 1a. Two specimens of the sandwich panel are positioned between the three rigid steel plates and glued together. The force $F$ acts on the middle plate and vertical displacement $w$ of the rigid plate is measured. Shear modulus $G_{C}$ is determined according to formulae:

$$
G_{C}=\frac{F}{2 \gamma B L}
$$

where shear deformation $\gamma=w / D$ and $B, L$ denote width and length of the sandwich specimens, respectively.

a)

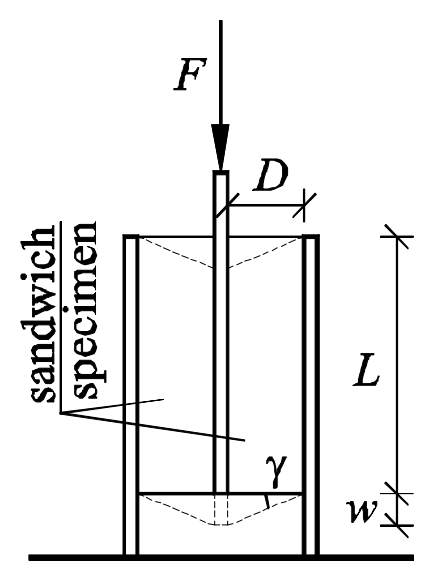

b)

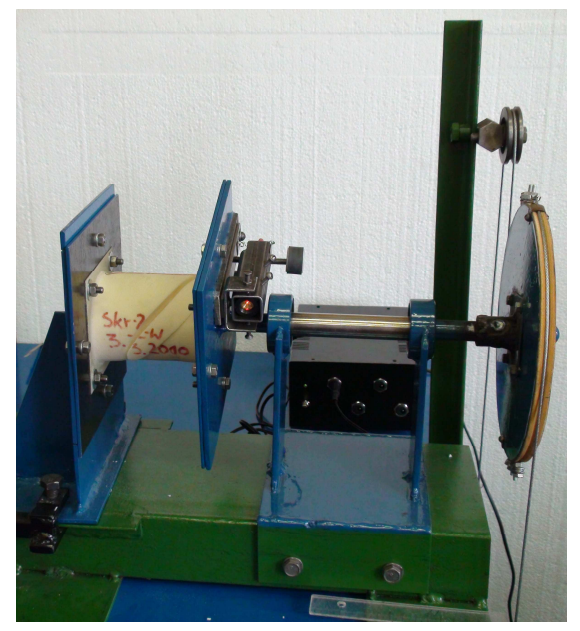

Fig. 1. Assessment of shear modulus: a) a scheme of the double-lap shear test, b) a torsion test

The second method of determination of Kirchhoff's modulus is torsion of cylindrical specimen. The mechanical equipment for this test is presented in Figure $1 \mathrm{~b}$. Torsion moment $M_{S}$ and angle of specimen rotation $\varphi$ are measured and the shear modulus is calculated using classical relation: 


$$
\varphi^{\prime}=\frac{M_{S}}{G_{C} I_{o}}
$$

where $I_{o}$ is a central second order moment of the area of the cylinder cross-section. The first derivative of the angle of rotation is known because $\varphi^{\prime}=\varphi / L(L-$ a length of the sample).

The ES recommends the shear of the beam test to determine $G_{C}$. It is worth noticing that the shear effect always coincides with the bending effect, but the decreasing of the beam length results in the decreasing of the bending effect. Therefore, a test on a short panel is called 'shear test', whereas a test on long panels is called 'bending test'. From the mathematical point of view [8], the behaviour of a short or a long flat sandwich panel is described by differential equations for vertical deflection $w$ and shear strain $\gamma$ :

$$
\begin{gathered}
w^{I V}=\frac{q}{B_{S}}-\frac{q^{\prime \prime}}{G_{C} A_{C}}, \\
\gamma^{\prime \prime}=-\frac{q^{\prime}}{G_{C} A_{C}},
\end{gathered}
$$

where $w, \gamma$ and distributed transverse load $q$ are the functions of the position coordinate $x$. The $G_{C}, A_{C}$ and $B_{S}$ denote shear modulus, cross-sectional area of the core and bending stiffness of the panel, respectively. Using differential relations for beams $M^{\prime}=V, V^{\prime}=-q$ ( $M$ - bending moment, $V$ - shear force), the following constitutive equations are obtained:

$$
\begin{gathered}
M=B_{S} \cdot\left(\gamma^{\prime}-w^{\prime \prime}\right), \\
V=G_{C} A_{C} \cdot \gamma .
\end{gathered}
$$

Displacement $w$ can be decomposed into two components

$$
w=w_{M}+w_{V}
$$

which refer to the bending and shear effect. Since $w_{V}^{\prime}=\gamma$, then:

$$
\begin{aligned}
& M=-B_{S} w_{M}^{\prime \prime}, \\
& V=G_{C} A_{C} w_{V}^{\prime} .
\end{aligned}
$$

The procedure of shear modulus determination consists of the following steps: measurement of the load and total deflection $w$ (usually in the middle od the 
beam), analytical determination of $w_{M}$ from (8) and then assessment of $G_{C}$ using (9) and (7).

It should be emphasized that the reliability of the method depends on the level of correspondence between the theory, Eqs. (3)-(9), and behaviour of real structures. The tests on short panels seem to be more appropriate because of the shear effect domination, but from the other point of view the long specimens reflect more realistic conditions of sandwich panel usage.

\section{Analysis of the influence of core compression}

The scheme of the recommended shear test is also known as the four point bending test is presented in Figure 2. The system is loaded by concentrated force $F$. Hence, two equal forces $F / 2$ act on the beam at points $x=L / 3$ and $x=2 L / 3$. To find shear modulus $G_{C}$, force $F$ and the deflection in the middle of the beam (point C) should be measured. Using Eqs. (7)-(9) we solve the static system and find the deflections:

$$
\begin{aligned}
w_{V}(\mathrm{C}) & =\frac{F L}{6 G_{C} A_{C}}, \\
w_{M}(\mathrm{C}) & =\frac{23}{1296} \frac{F L^{3}}{B_{S}} .
\end{aligned}
$$

Hence, shear modulus $G_{C}$ is calculated using the formulae:

$$
G_{C}=\frac{F L}{6 A_{C}\left(w-w_{M}\right)} .
$$

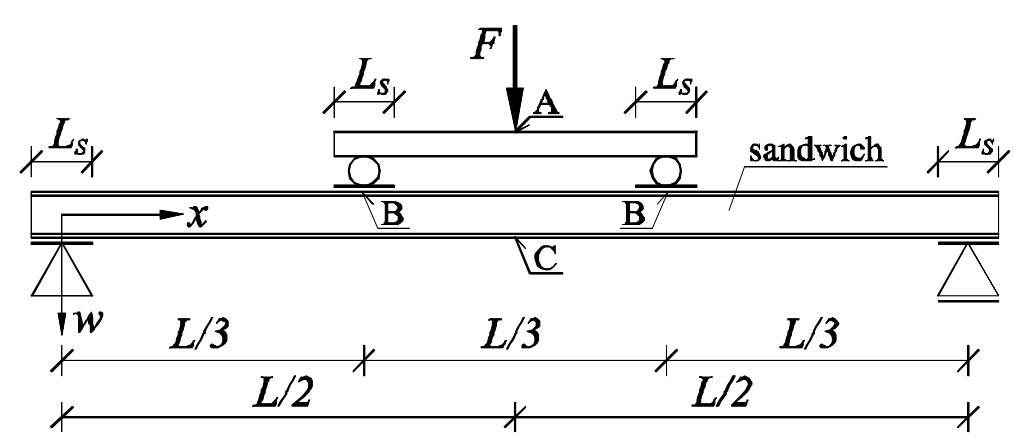

Fig. 2. The scheme of the shear test (four point bending test) 
The results of real four point bending tests indicate distinct dependence on the depth and the span of the panel. Looking for the reason of such unaccountable phenomenon, the consecutive three problems are discussed below.

\section{Problem 1}

Very often the displacement in the middle of the beam (point $C$ ) is wrongly considered as the displacement of the loading apparatus (point A). In fact, the displacements in point A are represented by:

$$
\begin{gathered}
w_{V}(\mathrm{~A})=w_{V}(\mathrm{~B})=\frac{F L}{6 G_{C} A_{C}}, \\
w_{M}(\mathrm{~A})=w_{M}(\mathrm{~B})=\frac{5}{324} \frac{F L^{3}}{B_{S}} .
\end{gathered}
$$

The bending part (14) is different from (11), but the shear part is the same. The change of $G_{C}$ result caused by this error can be calculated as:

$$
d G_{C 1}=\frac{F L}{6 A_{C}\left(w(\mathrm{~B})-w_{M}(\mathrm{C})\right)}-G_{C},
$$

where $G_{C}$ denote proper value of the shear modulus.

\section{Problem 2}

The concentrated forces acting on the beam at $x=L / 3$ and $x=2 L / 3$ compress the core. Simple relations for the compression stress and for the core compression have been assumed in the form:

$$
\begin{gathered}
\sigma_{C c}(\mathrm{~B})=\frac{F / 2}{\left(L_{S}+k D\right) B}, \\
w_{C c}=\sigma_{C c} D / E_{C c},
\end{gathered}
$$

where $L_{S}$ is the width of the load spreading plates, $D$ is the depth of the panel, $B$ is the width of the panel, $k$ is the distribution parameter (usually $k=0.5$ ) and $E_{C c}$ is modulus of elasticity of the core in compression. Taking into account the compression effect at the loading points $\mathrm{B}$, the initial value of $G_{C}$ changes as follows:

$$
d G_{C 2}=\frac{F L}{6 A_{C}\left(w(\mathrm{C})+w_{C c}-w_{M}(\mathrm{C})\right)}-G_{C} .
$$

In this case, the expression in denominator has only theoretical meaning and represents the shear part of the displacement in point $C$ increased by the effect of core compression at the loading points. The value $d G_{C 2}$ is presented only for comparison with the respective values described in Problem 1 and Problem 3. 


\section{Problem 3}

Similarly as in Problem 2 we can analyse the influence of the core compression at the end support. For the end support the compression stress has a different form

$$
\sigma_{C c}=\frac{F / 2}{\left(L_{S}+0.5 k D\right) B} .
$$

The change of the shear modulus with respect to end support compression $d G_{C 3}$ is expressed in the same form as $d G_{C 2}$. In this case, the expression $w(C)+w_{C c}$ in the denominator of (18) represents the most probable value of measured in reality displacement at point $\mathrm{C}$.

\section{Solution of the problems}

To illustrate the influence of the error described in Problem 1 and the influence of the core compression at the loading points (Problem 2) and at the end supports (Problem 3), the changes $d G_{C 1}, d G_{C 2}$, and $d G_{C 3}$ expressed by (15), (18) are presented in Figures 3-6 as the function of the span of the panel $L$ (with constant depth of the panel $D=0.06 \mathrm{~m}, D=0.16 \mathrm{~m}$ ) and as the function of depth $D$ (with constant span $L=1.0 \mathrm{~m}, L=3.0 \mathrm{~m}$ ). Initial value of $G_{C}$ is equal to $3000 \mathrm{kPa}$. The other parameters have the typical values: elasticity modulus of faces $E_{F}=210 \mathrm{GPa}$, thickness of both faces $0.5 \mathrm{~mm}, E_{C c}=4000 \mathrm{kPa}, L_{S}=0.06 \mathrm{~m}$. The functions $d G_{C}$ have a closed form and do not depend on $F$ and $B$.

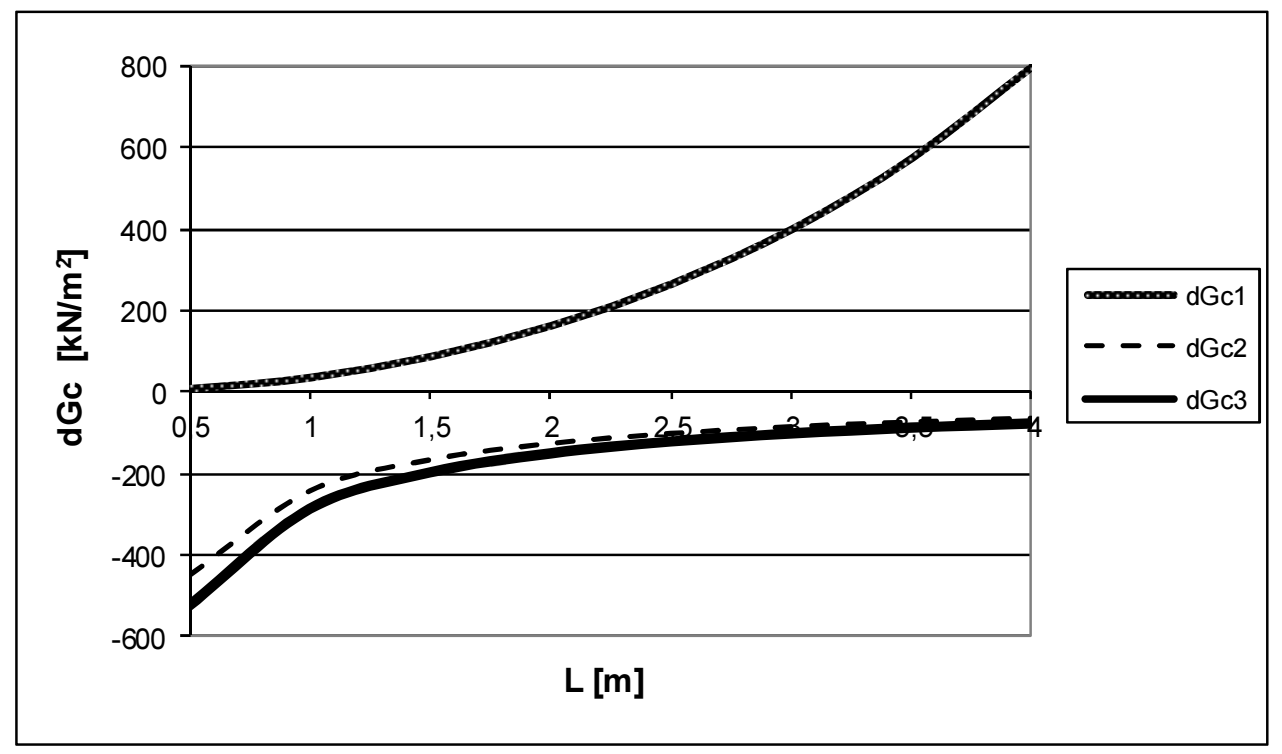

Fig. 3. The changes $d G_{C 1}, d G_{C 2}$ and $d G_{C 3}$ as the functions of the beam span $L$, $D=0.06 \mathrm{~m}$ 


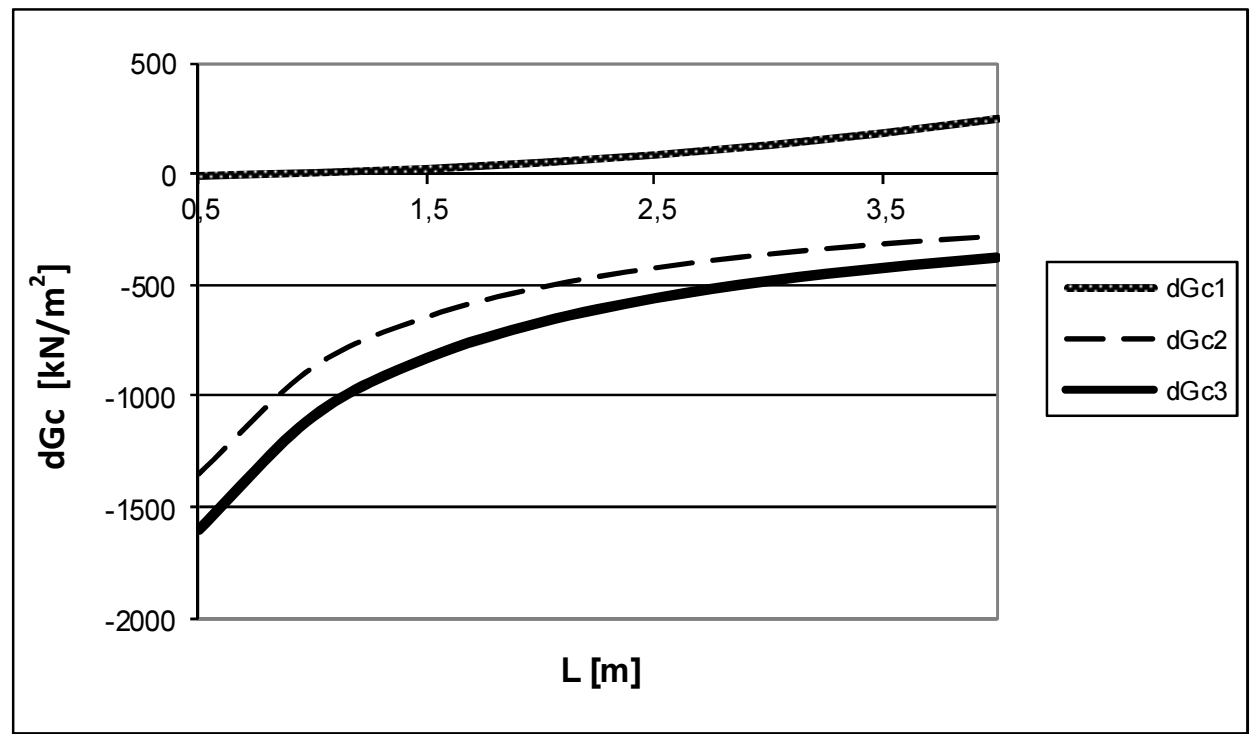

Fig. 4. The changes $d G_{C 1}, d G_{C 2}$ and $d G_{C 3}$ as the functions of the beam span $L$, $D=0.16 \mathrm{~m}$

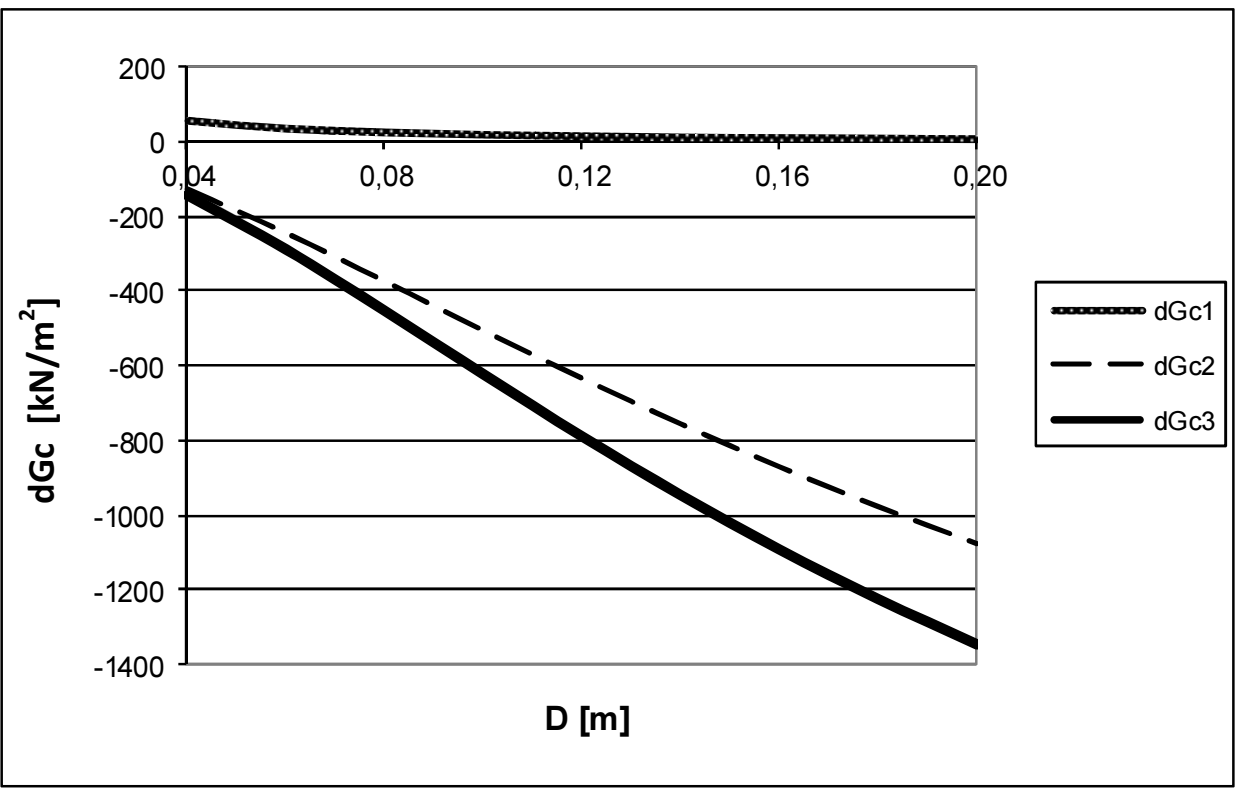

Fig. 5. The changes $d G_{C 1}, d G_{C 2}$ and $d G_{C 3}$ as the functions of the depth $D$, $L=1.0 \mathrm{~m}$ 


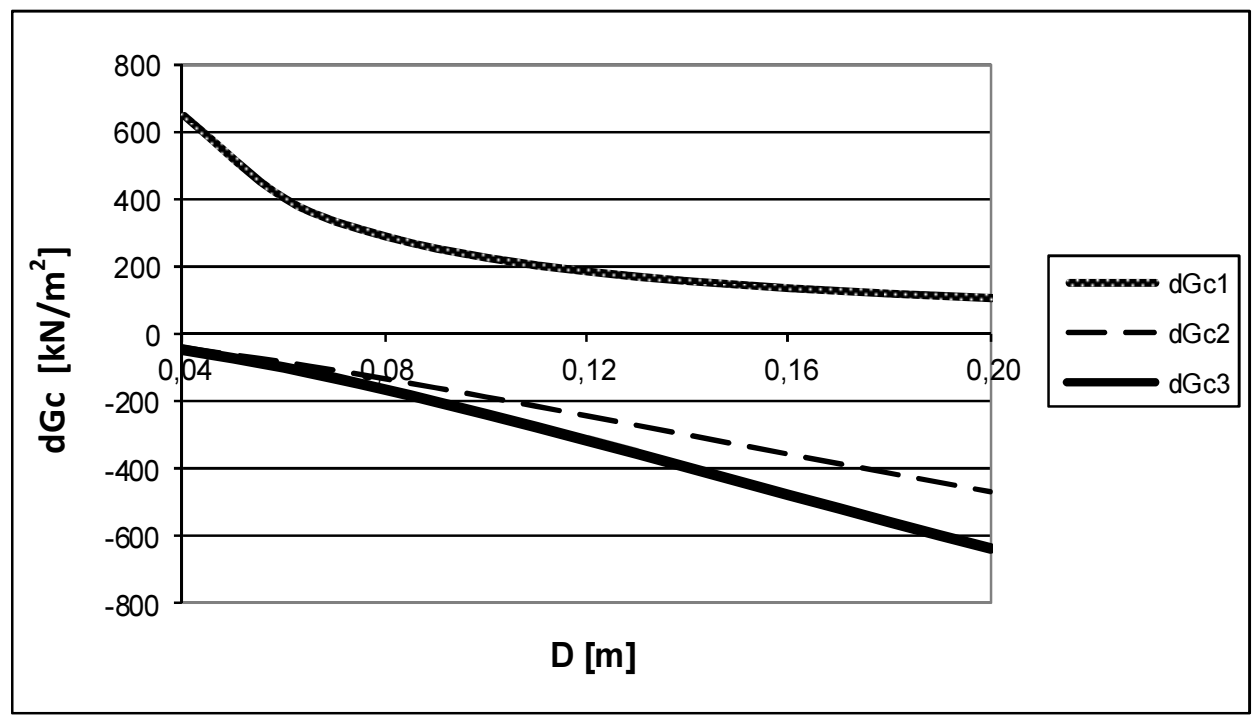

Fig. 6. The changes $d G_{C 1}, d G_{C 2}$ and $d G_{C 3}$ as the functions of the depth $D, L=3.0 \mathrm{~m}$

\section{Conclusions}

The presented analyses of the three mentioned problems show their high impact on the shear modulus assessment. Improper measurement of the deflection (Problem 1) for short panels increases shear modulus value about $2 \%$, but the significance of the error is obviously much higher for long panels.

The influence of the core compression is very important, i.e. if the compression at supports is neglected then the lower value of $G_{C}$ is measured. For example, for $L=1.0 \mathrm{~m}$ and $D=0.06 \mathrm{~m}$ the difference is equal to $288 \mathrm{kN} / \mathrm{m}^{2}$ which is almost $10 \%$ of the proper value $3000 \mathrm{kN} / \mathrm{m}^{2}$. In case of thicker core $(D=0.16 \mathrm{~m})$ the difference is higher and reaches $1092 \mathrm{kN} / \mathrm{m}^{2}$ (the value calculated using (12) for real structure, without any correction with respect to compression at supports, would be equal to $1908 \mathrm{kN} / \mathrm{m}^{2}$ ).

The paper shows that in the procedure of the shear modulus determination, the core compression effect shall be considered.

\section{References}

[1] Hoff N.J., Bending and buckling of sandwich beams, Journal of Aeronatical Sciences 1948, 15, $12,707-720$.

[2] Reissner E., Finite deflections of sandwich plates, Journal of Aeronatical Sciences 1948, 15, 7, 435-440.

[3] Bowles K.J., Vannucci R.D., Mechanical properties characterization of composite sandwich materials intended for space antennas applications, NASA Technical Memorandum 88893, 1986. 
[4] O'Connor D.J., An evaluation of test methods for shear modulus of sandwich cores, International Journal of Cement Composites and Lightweight Concrete 1984, 6, 3-12.

[5] Chuda-Kowalska M., Pozorski Z., Garstecki A., Experimental determination of shear rigidity of sandwich panels with soft core, Proc. of $10^{\text {th }}$ International Conference Modern Buildings Materials, Structures and Techniques, Vilnius, Lithuania, 2010, 56-63.

[6] EN 14509:2010 Self-supporting double skin metal faced insulating panels - Factory made products - Specifications, 2010.

[7] Kooistra G.W., Queheillalt D.T., Wadley H.N.G., Shear behavior of aluminum lattice truss sandwich panel structures, Material Science and Engineering 2008, 472, 242-250.

[8] Błaszczuk J., Pozorski Z., Engineering aspects of structural response of multi-span sandwich panels, Scientific Research of the Institute of Mathematics and Computer Science Czestochowa University of Technology 2010, 2(9), 5-15. 